\title{
Direct Current Stimulation alters neuronal input/output function
}

\author{
Belen Lafon, Asif Rahman, Marom Bikson, Lucas C. Parra
}

The City College of the City University of New York, Department of Biomedical Engineering, New York, USA

Corresponding author: Belen Lafon, The City College of The City University of New York, Department of Biomedical Engineering, 85 Saint Nicholas Terrace, 10027, New York, NY, USA.

Email: belulafon@gmail.com

Phone: 212.650 .8653

Abstract word count: 188 words

Word count: 4000 (excluding figure legends, abstract, keywords and references).

Funding: This work is supported by grants from the National Institute of Health (R01MH092926), NSF, and DoD (AFOSR).

Color print is required for this manuscript 


\section{Direct Current Stimulation alters neuronal input/output function}

\section{ABSTRACT}

Background: Direct current stimulation (DCS) affects both neuronal firing rate and synaptic efficacy. The neuronal input/output (I/O) function determines the likelihood that a neuron elicits an action potential in response to synaptic input of a given strength. Changes of the neuronal I/O

function by DCS may underlie previous observations in animal models and human testing, yet have not been directly assessed.

Objective: Test if the neuronal input/output function is affected by DCS

Methods: Using rat hippocampal brain slices and computational modeling, we provide evidence for how DCS modulates the neuronal I/0 function.

Results: We show for the first time that DCS modulates the likelihood of neuronal firing for a given and fixed synaptic input. Opposing polarization of soma and dendrite may have a synergistic effect for anodal stimulation, increasing the driving force of synaptic activity while simultaneously increasing spiking probability at the soma. For cathodal stimulation, however, the opposing effects tend to cancel. This results in an asymmetry in the strength of the effects of stimulation for opposite polarities.

Conclusions: Our results may explain the asymmetries observed in acute and long term effects of transcranial direct current stimulation.

\section{KEYWORDS}

Input-output; tDCS; electrical stimulation; two-compartment model; excitability; DCS 


\section{INTRODUCTION}

Transcranial direct current stimulation (tDCS) is actively investigated as a tool for non-invasive brain stimulation. Across diverse applications the general goal is to modulate neuronal excitability. tDCS produces current flow across the brain from anode to cathode. Classical in vivo animal studies demonstrate an increase in neuronal activity (firing rate) in brain regions under the anode and a decrease in activity under the cathode [1]-[5]. These early results motivated neurophysiological experiments with tDCS in humans [6] and clinical trials [7] with largely similar conclusions on neuronal excitability. Recent studies in brain slices confirmed changes in firing rate at both a single cell and network level, and attributed these to modulation of somatic membrane potential induced by DCS [8], [9]. However, in vitro studies established that synaptic efficacy is also affected by DCS [10]-[12]. Changes in synaptic input during DCS confound the reported changes in firing rate from somatic membrane polarization because modulation of the synaptic input to a cell will indirectly influence neuronal spiking. Effects of DCS on oscillatory activity and synaptic plasticity[13]-[16] may be similarly confounded. How prior results are explained by changes in input vs. changes in output is thus an open and compelling question. Here we consider if the reported changes in neuronal activity induced by DCS are a result of changes in synaptic input or independent of synaptic input and directly driven by somatic polarization producing changes in firing rate.

Whether input and output are independently modulated may relate to the biphasic membrane polarization produced during DCS. A negative electrode proximal to the apical dendrite of a pyramidal cell hyperpolarizes the soma and depolarizes apical dendrites ("cathodal” stimulation). In contrast, a positive electrode will depolarize the soma and hyperpolarize apical dendrites ("anodal" stimulation) [10], [17]. This biphasic polarization raises an important concern: how do 
synaptic inputs influence spike generation when the soma has the opposite polarization from the site of synaptic inputs (the apical dendrite)?

Here we use a combination of in-vitro experiments and computational modeling to address how DCS jointly modulates synaptic efficacy (input) and the likelihood of eliciting an action potential (output). Since we aim at a cellular description of these phenomena we use the hippocampal pyramidal cells as a model system for DCS effects, as these have been most extensively characterized [9]-[11], [13]-[16]. We hypothesized that DCS alters the I/O function, such that the synaptic input necessary to elicit an action potential is changed. We find that soma-depolarizing fields cause a leftward-shift of the I/O function, i.e. firing is increased for a fixed synaptic input. A computational neuron model suggests a synergistic combination of two effects: (a) dendritic hyperpolarization results in increased synaptic driving force leading to a bigger EPSP; and (b) somatic depolarization directly increases likelihood of firing. Thus, despite opposite polarization in soma and dendrite the effects of fields on input and output both point in the same direction, namely, increased firing for "anodal" stimulation and decreased firing for "cathodal" stimulation. However, we find that the effect is not symmetric with "cathodal" stimulation having a relatively weaker effect. This is explained by the computational model as a result of dendritic spike generation shift, which tends to cancel the suppressive effect of cathodal stimulation on somatic excitability. In summary, anodal stimulation has a synergistic effect on somatic and dendritic compartments whereas under cathodal stimulation the effects on the two compartments tend to cancel each other. Our results provide a possible cellular explanation to the asymmetries often found in animal and human tDCS experiments [10], [13], [18]-[26].

\section{MATERIALS AND METHODS}




\section{Ethical approval}

All animal experiments were carried out in accordance with guidelines and protocols approved by the Institutional Animal Care and Use Committee at The City College of New York, CUNY.

\section{Electrophysiology}

\section{Slice preparation}

Hippocampal slices were obtained from young male Wistar rats, aged 3-5 weeks old. Animals were anesthetized with ketamine (7.4 mg kg-1) and xylazine (0.7 mg kg-1) and sacrificed by cervical dislocation. The brain was removed and submerged in cutting solution containing (in $\mathrm{mM}$ ): $\mathrm{NaCl}$, 87; KCl, 2.5; $\mathrm{MgSO}_{4}, 7 ; \mathrm{NaH}_{2} \mathrm{PO}_{4}, 1.25 ; \mathrm{NaHCO}_{3}$, 25; D-glucose, 25; Sucrose, 75; $\mathrm{CaCl}_{2}, 0.5$; bubbled with carbogen $\left(95 \% \mathrm{O}_{2}, 5 \% \mathrm{CO}_{2}\right)$. Sagittal slices $(400 \mu \mathrm{m})$ were cut using a vibrating microtome and maintained at $4^{\circ} \mathrm{C}$. Slices were transferred to a room temperature solution constituted by $50 \%$ cutting solution and 50\% artificial cerebrospinal fluid, containing (in $\mathrm{mM}$ ): $\mathrm{NaCl}, 125 ; \mathrm{KCl}, 2.5$; $\mathrm{MgSO}_{4}, 1.5 ; \mathrm{NaH}_{2} \mathrm{PO}_{4}, 1.25 ; \mathrm{NaHCO}_{3}, 25 ; \mathrm{D}$-glucose, $25 ; \mathrm{CaCl}_{2}, 2$. After 30 min slices were placed in a pure ACSF solution for an hour before being placed in a fluid-gas interface chamber perfused with ACSF at $(30 \pm 1)^{\circ} \mathrm{C}$.

\section{Uniform constant electric fields}

Uniform "DC" fields were generated by passing constant current between two parallel Ag-AgCl wires positioned parallel to the direction of fluid flow (Figure 1A). Fields were applied with Ag-AgCl wires $11.2 \pm 0.4 \mathrm{~mm}$ long and placed $6.0 \pm 0.4 \mathrm{~mm}$ apart. We define anodal and cathodal stimulation consistent with the polarization profile induced in neurons. "Anodal" stimulation refers to when the negative electrode is closer to the alveus side of CA1, negative electric fields designate anodal 
direct current stimulation (-DCS). Positive direct current stimulation (+DCS) refers to "cathodal" stimulation (Figure 1A). $35 \mathrm{~V} / \mathrm{m}$ extracellular fields were applied for 1 second and orthodromic stimulation delivered 0.5 seconds after DCS started. The electric field intensity was chosen based on pilot experiments to produce a $5-10 \%$ change in extracellular field potentials amplitude such that a statistically significant change could be observed within slices with a practical sample size. During DCS the voltage varies linearly between parallel stimulation wires (Figure 1A). Experimental recordings show some fluctuation of the voltage in the horizontal direction. Prior to each experiment we assured that gradients in horizontal direction were less than $5 \%$ of the gradients along vertical direction. When tDCS is applied, current flows in the brain generating electric fields of different intensities, but at the length scale of a single cell the electric fields can be considered spatially uniform [27].

\section{Orthodromic pulsed stimulation}

Extracellular field potentials were recorded in hippocampal slices using microelectrodes (glass micropipettes filled with 0.25 M NaCl, resistance 1-6 MOhms). Orthodromic stimulation pulses were applied at Schaffer collateral with a platinum/stainless steel bipolar electrode placed 100-400 $\mu \mathrm{m}$ away from the recording site. The slice is carefully located so that the pre-synaptic fibers are perpendicular to the direction of the field, therefore avoiding possible effects of DCS polarizing presynaptic axon terminals [10]. Synaptic input was elicited every $30 \mathrm{~s}$ with a $0.2 \mathrm{~ms}$ monophasic pulse. Orthodromic stimulation intensity was set to elicit a $25-50 \%$ of the maximum response. Field excitatory postsynaptic potentials (fEPSPs), which quantify synaptic current entering a population of neurons, were measured at the lower layer of stratum radiatum in CA1(Figure 1A). Strength of the fEPSPs was measured as the maximal slope during the descending phase of the fEPSP (Figure 1B). Relative fEPSP change was calculated as: DCS-slope/control-slope, averaged over 30 trials per 
slice (In this initial experiment we chose a larger number of trials as in the subsequent experiments). The same analysis was done with fEPSP amplitudes with similar results (data not shown). Population spike (PS) reflects the number of synchronously firing cells [28]. PS was measured at stratum pyramidale layer in CA1 region (Figure 1A). Amplitude of PS was defined as the difference between the negative peak and the mean of the two flanking peaks (Figure 1B). Variations in PS were quantified as: DCS-amplitude/control-amplitude, averaged across 3 trials per slice.

\section{Neuronal input-output curves}

Following stable baseline recordings for 10 minutes, the input-output function was measured at CA1. Activity was simultaneously recorded from stratum radiatum and stratum pyramidale in CA1 (Figure 1A). The I/O curves were constructed by plotting PS amplitude (output) versus fEPSP slope (input) across the range of orthodromic stimulation intensities (300-500 $\mu \mathrm{A}$, Figure 1C). Orthodromic stimulation was applied every 30 seconds while simultaneously applying electric fields (+DCS or -DCS), or no field (control). Pulsed DCS was delivered in the following order: control, +DCS, control, -DCS; the polarities were randomly assigned so that half of the experiments started with +DCS and half with -DCS. Each sequence was repeated 3 times and the mean values were used for assessing the I/O curve.

\section{Data acquisition and analysis}

Analog signals were low-pass filtered $(3000 \mathrm{~Hz}$ cutoff, A-M Systems, WA, USA) and digitalized at a $10 \mathrm{kHz}$ sampling rate (Powerlab 16/35, AD instruments). Traces are aligned across trials by this sampling/stimulation equipment. Data is presented as mean \pm standard error of the mean, unless otherwise stated. 
Parameters of the I/O curve were determined by fitting the sigmoid curve: $f(x)=\frac{y_{\max }}{1+\exp \left[\left(x_{50}-x\right) / s\right]}$, $y_{\max }$ indicates the maximum $P S$ value, $x_{50}$ indicates the fEPSP value at which half of this maximum PS value is reached and $s$ (also called gain) is the slope of the sigmoid at $x_{50}$. Parameters in DCS conditions are normalized by the corresponding control condition and the deviation is calculated (Figure 3C). Statistical analyses of the parameters were done using two-tailed, one-sample Student's t tests to determine significant differences from zero.

\section{Two-compartment computational neuron model}

Uniform electric fields induce a change in membrane polarization across the cell [10], [17]. Neuronal compartments at opposing ends of a cell (along the direction of the field) experience opposite changes in membrane potential. The simplest model that can account for compartment specific polarization along the soma-dendrite axis is a two-compartment model [29], [30]. We used a reduced model of a pyramidal cell [31]-[33] with two electrically coupled compartments: one describing the dendrites and the other the somatic cell body and the axonal initial segment. Both compartments are described by active and passive conductances following Hodgkin Huxley formalism. All the channel dynamics and transition rates are described in [31], [32], [34], [35]. Figure 2D shows a schematic representation of the model as well as its coupling to the extracellular field through the extracellular potential difference $V_{\mathrm{E}}$. The details and equations of the model can be found in the supplementary material.

\section{RESULTS}

During tDCS an extracellular field is generated in the brain affecting cortical and subcortical structures including deep brain structures such as the hippocampus [36], also affecting 
hippocampus-dependent tasks [37], [38]. Local stimulation can be recreated in vitro by applying uniform electric fields across hippocampal slices, which allow micro-recording from the dendritic and somatic layers independently (Figure 1A). Field excitatory post-synaptic potentials (fEPSP) measured at the dendrites reflects the aggregate post-synaptic current to a population of neurons; which provides an accurate measure of the synaptic input (Figure 1B). Population spikes (PS) are measured in the somatic layer, registering the summed spiking activity of a group of neurons; the higher the relative amplitude of the PS the greater the number of synchronously firing cells for that population of neurons (Figure 1B). Increasing the orthodromic stimulation intensity increases synaptic input (fEPSP), which in turn increases spiking output (PS). Sweeping across increasing orthodromic stimulation intensities results in a curve of synaptic input versus spiking output that defines the input-output function (Figure 1C). The I/O function determines spiking output for a given synaptic input strength, thus quantifying the responsiveness of neurons.

Previous studies have shown that synaptic input can be affected by DCS [10]-[12], modulating synaptic current flow into neurons, which can produce a corresponding change in firing level. Note that this modulation of the input does not necessarily affect the responsiveness of a neuron; the I/O curve may be unchanged with no alteration of how much neuronal firing is produced in response to a given synaptic input (Figure 1D). Alternatively, a change in synaptic input can be supplemented with an amplified firing response, thus inducing a shift of the I/O function (Figure 1E); neurons are now in a more responsive state to a given synaptic input. In essence we ask whether modulation of overall excitability is the result of only a strengthened synaptic input (Figure 1D), or can be supplemented by increased responsiveness for a fixed input (Figure 1E). 


\section{Direct current stimulation modulates synaptic input and neuronal output}

First, we characterized the effects of DCS on field excitatory postsynaptic potential (fEPSP) and population spike (PS) separately. Field EPSP and PS were recorded from the hippocampal CA1 area during DCS. Changes in fEPSP slope induced by DCS are calculated as the ratio between the slope during DCS versus control (Figure 2A). Negative DCS $(-35 \mathrm{~V} / \mathrm{m})$, which depolarizes the soma and hyperpolarizes the apical dendritic tree, significantly increased fEPSP slope $(8.23 \pm 4.44 \%, \mathrm{p}<0.05$, $\mathrm{n}=17)$. Positive DCS $(+35 \mathrm{~V} / \mathrm{m})$, which hyperpolarizes the soma and depolarizes apical dendrites, decreased fEPSP slope $(-14.05 \pm 4.31 \%, \mathrm{p}<0.05, \mathrm{n}=13)$. Analysis was done on fEPSP slopes but similar results are obtained for fEPSP amplitudes (data not shown). At the intensities and durations tested, DCS did not have any significant long lasting effects on fEPSP slopes, which recovered to baseline within 30 seconds. Fiber volleys from Schaffer Collaterals were also measured during DCS with no significant modulation during DCS (root-mean square of fiber volleys did not differ between DCS and control, $\mathrm{p}>0.05$, tested on $\mathrm{n}=11$ slices). Thus ruling out the possibility that DCS affects the number of fibers being activated during orthodromic stimulation, which is also not expected when the direction of the field is perpendicular to the pre-synaptic fibers [12].

These experimental results were replicated using a computational model of a two-compartment neuron (Figure 2C). The model has a somatic and dendritic compartment and is coupled to the extracellular field stimulation via an extracellular voltage gradient $\mathrm{V}_{\mathrm{E}}$ following Park et al., 2005 [29]. It differs from earlier models only in that the dendritic compartment can also initiate sodium spikes consistent with previous literature [39]-[46]. The membrane polarization induced in each compartment by positive and negative DCS is shown in Figure 2E, the polarization in the somatic compartment is greater than in the dendritic, in agreement with previous experimental recordings in pyramidal neurons in CA1 [10]. The change in EPSP slope in the model during DCS was 
modulated in opposite directions with field polarity, consistent with extracellular recordings of the field EPSPs (Figure 2C). The difference in magnitude between model and experiment may be due to a scaling factor between single neuron and population behavior and dynamics.

Prior literature has conflicting results on the effect of DCS on fEPSP slope, and one possibility is that it depends on the direction of the activated fiber bundles [10]-[12]. To test for a dependence on the direction of propagation of afferent volleys, we stimulated Schaffer collateral fibers while recording laterally within stratum radiatum on opposite sides of the stimulating electrode (Figure 2F). Negative DCS enhanced and positive DCS inhibited fEPSP slope in both locations. A two-factor analysis of variance showed a significant effect for polarity $(F(1,12)=5.66, p<0.05)$ but no effect for recording locations $(F(1,12)=4.38, p>0.05)$ and no interaction $(F(1,12)=0.30, p>0.05)$, i.e. the fEPSP was similarly modulated with polarity independently of the direction of AP propagation. The change in fEPSP at the proximal or distal location was correlated $\left(\mathrm{r}^{2}=0.57\right)$. Combined, these results indicate that the effect of DCS on synaptic efficacy does not depend on the direction of AP propagation, at least when the direction of the field is perpendicular to the orientation of the fibers stimulated orthodromically.

The spiking output, measured extracellularly as the population spike (PS), was assessed in the presence of DCS in stratum pyramidale (Figure 2B). Negative DCS (-35 V/m) significantly increased PS amplitude $(31.08 \pm 11.18 \%, \mathrm{p}<0.05, \mathrm{n}=15)$ and positive DCS $(+35 \mathrm{~V} / \mathrm{m})$ decreased PS amplitude $(-$ $11.25 \pm 6.36 \%, \mathrm{p}>0.05, \mathrm{n}=15$ ). The change in spiking output by DCS was reproduced by the model (Figure 2D). The firing level was measured by running the model with random synaptic input values fluctuating around some mean value (described in methods), and the number of spikes elicited during DCS is normalized by the number of spikes in the control condition. These results on firing activity indicate a polarity-specific effect of DCS on neuronal firing in agreement with 
previous findings [10], [13]. However, as shown above, the synaptic drive is also modulated by electric fields and therefore may have an indirect effect on neuronal firing in addition to the direct effect that DCS-induced membrane polarization has on firing.

\section{Direct current stimulation modulates the neuronal input-output function}

To disambiguate the relative contributions of synaptic effects at the dendrite from those of action potential generation at the soma we determined the neuronal input-output function for the computational model as well as in brain slice. In the model the spiking output is quantified as the

firing probability and the synaptic input as the average EPSP slope across the group of neurons (see supplementary methods). In the model membrane polarization induced by DCS modified the threshold sensitivity $\left(x_{50}\right.$, in the fitted model) in firing probability (Figure 3A). Negative DCS $(\sim 4.2$ $\mathrm{mV}$ polarization) produced a leftward shift of $-21.63 \%$ in the $\mathrm{I} / \mathrm{O}$ function compared to control. Conversely, positive DCS produced a rightward shift of $1.53 \%$ (Figure 3C). The change in gain (slope of the sigmoid at $\mathrm{x}_{50}$ ) for negative and positive DCS is $-3.74 \%$ and $36.10 \%$ respectively. Note that the horizontal asymptote for single-neuron simulations of the input-output function represents the firing probability, therefore the maximum value is always 1 .

In the slice preparation, spiking output is measured as PS amplitude and synaptic input is captured by fEPSP slope. Experimentally recorded input-output function also shows a lateral shift with DCS in the same direction as the model (Figure 3B,C). In accordance with the model, the required synaptic input (fEPSP) to elicit $50 \%$ of population firing $\left(x_{50}\right)$ is shifted by $-30.31 \pm 5.48 \%(\mathrm{p}<0.05$, $\mathrm{n}=15)$ for negative DCS and $5.46 \pm 3.11 \%(\mathrm{p}<0.05, \mathrm{n}=15)$ for positive DCS. There is no significant change in gain (-DCS: 10.45 $\pm 12.78 \%$; +DCS: 13.52 $\pm 5.05 \%, \mathrm{n}=15)$. The horizontal asymptote shows a significant modulation with positive and negative DCS of $6.72 \pm 2.02 \%$ and $-10.31 \pm 1.69 \%$, 
respectively. Figure 3D shows increased neuronal firing for given synaptic input (fixed fEPSP amplitude). Note that fEPSPs with the same magnitude produce quite different population spikes for different DCS polarities $(-35 \mathrm{~V} / \mathrm{m}: 1.7 \mathrm{mV}$; +35 V/m: $0.26 \mathrm{mV}$; control: $0.43 \mathrm{mV})$. Thus, the model and experimental data indicate that a subthreshold synaptic input can become suprathreshold in the presence of soma depolarizing and dendrite hyperpolarizing electric field (DCS).

We observed that positive DCS is less effective in modulating $x_{50}$ than negative DCS. How can this asymmetry in responsiveness of the neuron to DCS be explained? Negative DCS depolarizes the soma, thus increasing firing likelihood. In addition it hyperpolarizes the dendrite, which increases the drive for synaptic currents and thus increases EPSP magnitude. In contrast, positive DCS hyperpolarizes the soma and depolarize the dendritic membrane, increasing the probability of spike initiation at the latter location. The model suggests that when the soma is hyperpolarized the action potential can be triggered at the dendrite, therefore preventing a reduction in firing by positive DCS. This shift to dendritic spike initiation may already have been apparent in earlier experimental data (see Figure 5 and 6 from Bikson et al., 2004). It is important to note that we could not replicate this phenomenon unless we incorporated active sodium channels into the dendritic compartment (see Figure S1, supplementary material). In the absence of active channels in the dendrite, the depolarized dendrite diminishes EPSP strength and the firing is suppressed in the hyperpolarized soma resulting in an equally strong suppressive effect for positive DCS. In summary, there is an asymmetric shift in the experimental I/O function that results from a synergistic effect for negative DCS but a cancellation of effects for positive DCS that is only apparent with active dendritic conductances 


\section{Direct current stimulation differentially modulates spike time.}

Neurons convey information not only through their firing rate but also with the timing of individual spikes. To investigate how DCS affects firing time we measured changes in latency in the computational model as well as in the experimental data. Comparing the firing time of the population spike measured in slices with the two-compartment neuron model spiking times. The difference in spike timing between control and DCS conditions, $\Delta T$, was measured in the model across different orthodromic stimulation intensities (Figure 4A). Negative DCS induces a mean $\Delta T$ of $-2.82 \mathrm{~ms} \pm 0.32 \mathrm{~ms}$ (averaged over intensities) and $1.26 \mathrm{~ms} \pm 0.31 \mathrm{~ms}$ for positive DCS. Negative and positive $\Delta \mathrm{T}$ represent an advancement or delay of firing respectively. In the experiment $\Delta \mathrm{T}$ was measured as the modulation in latency of the population spike for different orthodromic stimulations intensities and again averaged over intensities (Figure 4B). -DCS induced a significant mean $\Delta \mathrm{T}$ of $-0.31 \mathrm{~ms} \pm 0.07 \mathrm{~ms}(\mathrm{p}<0.05, \mathrm{n}=15)$ and $+\mathrm{DCS}$ resulted in a mean $\Delta \mathrm{T}$ of $0.14 \mathrm{~ms} \pm 0.04 \mathrm{~ms}$ $(\mathrm{p}<0.05, \mathrm{n}=15)$. The direction of change in firing time is in agreement with the model, as well as the asymmetry in effect size.

\section{Considerations for the modulation of I/O by DCS in the human cortex}

During tDCS, current flows from anode to cathode. Conventionally, it is thought the area under the anode experiences an increase in excitability, whereas the opposite happens under the cathode. How the neuronal I/O function mediates these changes in excitability has not been addressed yet. Here we use the computational model to predict how electric fields generated by tDCS could affect the I/O function in the presence of the electric field distributions expected for the human cortex. 
First we examined how field orientation and magnitude modulates the I/O function in the neuron model (Figure 5A). The simulation predicts that the horizontal shift of the I/O function is monotonic with field intensity, in accordance with previous physiological results [10], [12], resulting in approximately a $-0.45 \%$ shift per $\mathrm{V} / \mathrm{m}$ applied for -DCS and $0.04 \%$ per V/m for +DCS (Figure 5 A.1). Orientation of the electric field with respect to the somato-dendritic axis will determine the polarization of the neuronal membrane. Under perfect alignment (Figure 5A.2) the somatic cell membrane and the dendritic compartment are maximally polarized, resulting in the greatest horizontal shift of the I/O function. When the field is perpendicular to the neuronal axis the cell body and dendrites are minimally polarized (Figure 5A.4), resulting in no change of the I/O function compared to control. For each orientation (Figure 5A.2-4), the field was treated as a vector and decomposed into the tangential and radial (somato-dendritic axis) components. The radial component is the effective field that induces membrane polarization in the neuron.

When tDCS is applied in humans, orientation and intensity of the field are expected to vary across the brain [12], [47]. However, tangential current flow will not affect the I/O function (Figure 5A.4). In 2013, Rahman et al. analyzed what fraction of cortical tissue is exposed to tangential current flows versus 'radial' flows, i.e. along the somato-dendritic axis of pyramidal neurons. We repeated this analysis for the conventional montage of electrodes M1-SO (Figure 5B.1) which results in radial fields with distribution shown in Figure 5B.2. Neurons in the cortical gyri will experience soma hyperpolarization and depolarization according to their location relative to the current flow. For these neurons the effects of the I/O was simulated and averaged, resulting in the aggregate I/O effect for the population of neurons (Figure 5B.3). We calculated the combined effect for a group of neurons by sampling a 100 times from the probability density function of the electric field underneath the anode and the cathode (Figure 5B.4). There is a mix of polarization in the areas 
underneath the electrodes, with a predominance of negative fields beneath the anode (soma depolarizing) and vice versa for the cathode. Thus the aggregate I/O underneath the anode is subject to a leftward shift of the I/O (-0.05\% shift per V/m applied) (Figure 5B.5, 5B.6). For the cathode the sensitivity of the population $\mathrm{I} / \mathrm{O}$ is smaller $(0.01 \%$ change per $\mathrm{V} / \mathrm{m}$ applied $)$ due to the asymmetry described previously (Figure 3).

Under the electrodes, neurons experience variable intensities of fields (Figure 5B.4), the aggregate effect on the I/O is dominated for the neurons that experience a very small field (most cells undergo fields near $0 \mathrm{~V} / \mathrm{m}$ ). Thus producing a very small shift of the aggregate I/O curve under the anode/cathode when compared to results obtained in Figure 5A: $-0.05 \% / 0.01 \%$ per V/m under the anode/cathode (Figure 5B.6) versus $-0.45 \% / 0.04 \%$ per $\mathrm{V} / \mathrm{m}$ for experimental and modeling data in hippocampal brain slices (Figure 5A.1, Figure 3C). We calculated the rate of change of the I/O under the anode/cathode for neurons that are in the 95th percentile of the electric field distribution, meaning the ones that experience the peak electric field (Figure 5B.4, 5B.7). Under the anode the $\mathrm{I} / \mathrm{O}$ function of the neurons that are maximally depolarized at the soma changes $-0.2 \%$ per $\mathrm{V} / \mathrm{m}$ and $0.02 \%$ per $\mathrm{V} / \mathrm{m}$ for the cells with topmost soma hyperpolarization. Under the cathode the predicted modulation is $-0.1 \%$ per $\mathrm{V} / \mathrm{m}$ and $0.06 \%$ per $\mathrm{V} / \mathrm{m}$ respectively. Despite mixed polarization of the cells under the anode, excitability changes are dominated by neurons with depolarized soma resulting in increased excitability. Whereas under the cathode excitability reduction is comparable to its increase, meaning that the cells that are mostly depolarized and hyperpolarized under the cathode experience changes of the I/O of similar magnitudes but opposite direction $(0.06 \%$ per $\mathrm{V} / \mathrm{m}$ versus $-0.1 \%$ per $\mathrm{V} / \mathrm{m})$.

\section{DISCUSSION}


We demonstrate how under DCS the I/O function is changed, modifying the likelihood of firing for a fixed synaptic input strength. The I/O function determines whether a neuron fires or not, contributing to the neural processing within local circuits that ultimately affects neurophysiological and behavioral outcomes [48]. Overall DCS tends to shift the threshold of the I/O function making neurons more responsive to synaptic stimuli (Figure 1F), which is a crucial component in most of motor and cognitive tasks [49]-[52].

It is well established that DCS modulates synaptic efficacy (synaptic input) and population firing (spiking output), each as independent measures. Input measurements such as synaptic transmission or pre-synaptic activation are also modified during DCS [53]-[55] , and mixed effects have been reported for fEPSPs [10]-[12]. Output responses measured in animal models such as firing rate, network oscillation, excitability [8]-[10], [13], [57], [58]; and excitability measures in humans such as sensitivity to motor evoked potentials with transcranial magnetic stimulation [6], [59], [60] or visually evoked potentials are all modulated during DCS as well [61], [62]. However, the simultaneous modulation of firing activity and synaptic efficacy during DCS raises a basic question: how much of the previously observed changes in output (firing) are due to changes in synaptic current entering the cell as opposed to a change in the responsiveness of the cell?

Previous studies report that a whole-cell membrane depolarization induces a shift in the I/O function of neurons [63], [64]. Under DCS the polarization profile of neurons is more complex with opposite polarization along the somato-dendritic axis. During negative DCS ('anodal stimulation') current flows from the apical dendrites to the soma, hyperpolarizing the dendritic membrane and depolarizing the soma. The opposite occurs for +DCS ('cathodal stimulation'), the dendrites are depolarized and the soma hyperpolarized. The computational model suggests that opposing 
polarizations have a synergistic effect that leads to a leftward shift in the I/O threshold under anodal stimulation: First, by hyperpolarizing the membrane at the dendrites the driving force for synaptic current is increased following a simple and well-established relationship: $I_{\text {syn }}=g_{\text {syn }}\left(V_{m}-E_{\text {syn }}\right)$ [65], therefore increasing the synaptic current entering the cell [66]. Second, this increased synaptic input has a higher likelihood of producing an action potential given the depolarization at the soma induced by DCS. The interaction between the two compartments results crucial for describing the effects of DCS at a cellular level, encouraging the use of neuron models with two or more compartments in future studies.

Previous work by our group and others has focused on synaptic effects of DCS. Various groups have shown that fEPSP size is modulated by DCS [10]-[12], attributing a fraction of that to DCS effects on pre-synaptic axon terminals. Here we were interested in the $\mathrm{I} / \mathrm{O}$ function and specifically controlled for such pre-synaptic effects by using fields that are perpendicular to the axon bundle [12]. To verify that presynaptic strength was not modulated we measured the size of the fiber volley and found not effect of DCS. Note also that axonal effects do not directly impact the "output", as output is measured in terms of population firing (i.e number of spikes) and not the efficacy of each spike. Hence, the computational model here did not need to include an axonal compartment, but future work may want to combine the observed somatic, dendritic and axonal effects of DCS in a single computational model.

Positive DCS induces an opposite rightward shift of the I/O function but the magnitude of this shift is smaller compared to negative DCS. Figure 3D shows that the reduction of firing for the same fEPSP is less for +DCS than the augmentation produced by -DCS. Why is DCS stimulation more 'effective' for one polarity than the other? The computational model suggests that this asymmetry is due to the site of dendritic spike initiation. +DCS hyperpolarizes the soma and depolarizes the dendrite, therefore reducing the distance to the action potential threshold at the dendrites. When 
an orthodromic input arrives to the active dendrite it produces a sodium spike that will trigger a spike at the soma, in spite of its membrane hyperpolarization. Previous studies show that sodium spikes can be generated in the soma or dendrites depending on the pre-synaptic stimulus intensity and pattern [39]-[46]. In 2004, Bikson et al. measured a shift in spike initiation from the soma towards the dendrite under +DCS that resulted in an asymmetric modulation of firing.

Active dendrites may also explain the asymmetry observed for the timing effect of DCS. Negative DCS depolarizes the soma, which decreases the distance to threshold; therefore decreasing the time to spike [14]. With positive DCS fields the spike initiation moves to the dendrite and thus the expected delay from somatic hyperpolarization is reduced. Active conductances were critical in order for the model to fully reproduce the experimental results describing I/O modulation. While simpler models with passive dendrites were able to reproduce the direction of the I/O shift, they failed to reproduce the asymmetry (see supplementary material, Figure S1).

Although we have presented a possible explanation for the observed effects of DCS, a number of alternative mechanisms are also possible: 1) Differential effect of DCS on inhibitory and excitatory neurons due to their different morphologies, thus affecting the feed-forward inhibition circuit of cells in CA1 [67]-[69]. 2) Extracellular buffering of potassium by glial cells can affect intrinsic excitability of neurons [70]. Indeed, a recent study has shown a strong effect of DCS on glial function [71]. Future studies using patch-clamp recordings or calcium imaging measurements may provide direct support of the physiological mechanism proposed in this study.

There are important differences between the present in-vitro experiment with DCS and what may be expected with tDCS in human studies. One of important caveat is the fact that the orientation of the electric field relative to the somato-dendritic axis varies along cortical gyri and sulci. Field 
orientation is important as it will affect the sensitivity of the neurons to the field magnitude [72], by virtue of varying the induced membrane polarization (Figure 5). In the model, the effect of DCS on the I/O function is reduced whenever the field is at an angle from the somato-dendritic axis (Figure 5A.2-4). Another important difference between the present in-vitro experiments and electric fields expected for tDCS in humans is the intensity of the field. We have used strong fields to obtain robust experimental effects. To estimate effect sizes for smaller fields we simulated changes in the I/O function for various field intensities (Figure 5). The shift of the I/O scales monotonically with the intensity of the electric field and there is nothing in our results that suggests a lower threshold for the observed effects. We estimate a $0.6 \%$ change of the $\mathrm{X}_{50}$ threshold per $\mathrm{V} / \mathrm{m}$ applied for anodal stimulation. This compares to a $0.5 \%$ effect on firing rate [9] and a $1.1 \%$ effect on synaptic efficacy per $\mathrm{V} / \mathrm{m}$ applied [12].

Many animal and human studies have been explained or motivated by the notion of a change in neuronal excitability as a result of membrane polarization [6], [10], [13], [14], [17], [22], [73]. Until recently [12], the argument typically focused on somatic polarization of pyramidal cells, and tended to ignore the simultaneous opposite polarization of apical dendritites. The more detailed analysis provided here for the interaction of these two effects provides a possible explanation for the numerous findings that anodal and cathodal effects are not of equal strength in many human and animal studies [18]-[20], [24], [74], anodal often having a stronger effect [21]-[23], [25], [26]. The computational model suggests that the region under the anode has increased excitability, with a higher likelihood of firing for a given synaptic input. The region under the cathode has decreased excitability, but this reduction is smaller in magnitude when compared to the modulation of the I/O function under the anode, as often observed in human tDCS studies.

\section{ACKNOWLEDGEMENTS}


We greatly acknowledge Gregory Kronberg for helpful discussions and proofreading the

manuscripts.

\section{REFERENCES}

[1] L. J. Bindman, O. C. Lippold, and J. W. Redfearn, "Long-lasting changes in the level of the electrical activity of the cerebral cortex produced bypolarizing currents," Nature, vol. 196, pp. 584-585, Nov. 1962.

[2] O. D. Creutzfeld, G. H. Fromm, and H. Kapp, "Influence of transcortical d-c currents on cortical neuronal activity," Exp. Neurol., vol. 5, pp. 436-452, Jun. 1962.

[3] I. B. Gartside, "Mechanisms of sustained increases of firing rate of neurones in the rat cerebral cortex after polarization: role of protein synthesis," Nature, vol. 220, no. 5165, pp. 383-384, Oct. 1968.

[4] D. P. Purpura and J. G. Mcmurtry, "Intracellular activities and evoked potential changes during polarization of motor cortex," J. Neurophysiol., vol. 28, pp. 166-185, Jan. 1965.

[5] C. A. Terzuolo and T. H. Bullock, "MEASUREMENT OF IMPOSED VOLTAGE GRADIENT ADEQUATE TO MODULATE NEURONAL FIRING," Proc. Natl. Acad. Sci. U.S.A., vol. 42, no. 9, pp. 687-694, Sep. 1956.

[6] M. A. Nitsche and W. Paulus, "Excitability changes induced in the human motor cortex by weak transcranial direct current stimulation," J. Physiol. (Lond.), vol. 527 Pt 3, pp. 633-639, Sep. 2000.

[7] A. R. Brunoni, M. A. Nitsche, N. Bolognini, M. Bikson, T. Wagner, L. Merabet, D. J. Edwards, A. Valero-Cabre, A. Rotenberg, A. Pascual-Leone, R. Ferrucci, A. Priori, P. S. Boggio, and F. Fregni, "Clinical research with transcranial direct current stimulation (tDCS): challenges and future directions," Brain Stimul, vol. 5, no. 3, pp. 175-195, Jul. 2012.

[8] F. Fröhlich and D. A. McCormick, "Endogenous electric fields may guide neocortical network activity," Neuron, vol. 67, no. 1, pp. 129-143, Jul. 2010.

[9] D. Reato, A. Rahman, M. Bikson, and L. C. Parra, "Low-intensity electrical stimulation affects network dynamics by modulating population rate and spike timing," J. Neurosci., vol. 30, no. 45, pp. 15067-15079, Nov. 2010.

[10] M. Bikson, M. Inoue, H. Akiyama, J. K. Deans, J. E. Fox, H. Miyakawa, and J. G. R. Jefferys, "Effects of uniform extracellular DC electric fields on excitability in rat hippocampal slices in vitro," J. Physiol. (Lond.), vol. 557, no. Pt 1, pp. 175-190, May 2004.

[11] A. Y. Kabakov, P. A. Muller, A. Pascual-Leone, F. E. Jensen, and A. Rotenberg, "Contribution of axonal orientation to pathway-dependent modulation of excitatory transmission by direct current stimulation in isolated rat hippocampus," J. Neurophysiol., vol. 107, no. 7, pp. 18811889, Apr. 2012.

[12] A. Rahman, D. Reato, M. Arlotti, F. Gasca, A. Datta, L. C. Parra, and M. Bikson, "Cellular effects of acute direct current stimulation: somatic and synaptic terminal effects," J. Physiol. (Lond.), vol. 591, no. Pt 10, pp. 2563-2578, May 2013.

[13] J. G. Jefferys, "Influence of electric fields on the excitability of granule cells in guinea-pig hippocampal slices," J. Physiol. (Lond.), vol. 319, pp. 143-152, 1981.

[14] T. Radman, Y. Su, J. H. An, L. C. Parra, and M. Bikson, "Spike timing amplifies the effect of electric fields on neurons: implications for endogenous field effects," J. Neurosci., vol. 27, no. 11, pp. 3030-3036, Mar. 2007. 
[15] F. Ranieri, M. V. Podda, E. Riccardi, G. Frisullo, M. Dileone, P. Profice, F. Pilato, V. Di Lazzaro, and C. Grassi, "Modulation of LTP at rat hippocampal CA3-CA1 synapses by direct current stimulation," J. Neurophysiol., vol. 107, no. 7, pp. 1868-1880, Apr. 2012.

[16] D. Reato, M. Bikson, and L. C. Parra, "Lasting modulation of in vitro oscillatory activity with weak direct current stimulation," J. Neurophysiol., vol. 113, no. 5, pp. 1334-1341, Mar. 2015.

[17] C. Y. Chan, J. Hounsgaard, and C. Nicholson, "Effects of electric fields on transmembrane potential and excitability of turtle cerebellar Purkinje cells in vitro," J. Physiol. (Lond.), vol. 402, pp. 751-771, Aug. 1988.

[18] T. Elbert, W. Lutzenberger, B. Rockstroh, and N. Birbaumer, "The influence of low-level transcortical DC-currents on response speed in humans," Int. J. Neurosci., vol. 14, no. 1-2, pp. 101-114, 1981.

[19] F. Fregni, R. Marcondes, P. S. Boggio, M. A. Marcolin, S. P. Rigonatti, T. G. Sanchez, M. A. Nitsche, and A. Pascual-Leone, "Transient tinnitus suppression induced by repetitive transcranial magnetic stimulation and transcranial direct current stimulation," Eur. J. Neurol., vol. 13, no. 9, pp. 996-1001, Sep. 2006.

[20] P. Garin, C. Gilain, J.-P. Van Damme, K. de Fays, J. Jamart, M. Ossemann, and Y. Vandermeeren, "Short- and long-lasting tinnitus relief induced by transcranial direct current stimulation," J. Neurol., vol. 258, no. 11, pp. 1940-1948, Nov. 2011.

[21] A. Kraft, J. Roehmel, M. C. Olma, S. Schmidt, K. Irlbacher, and S. A. Brandt, "Transcranial direct current stimulation affects visual perception measured by threshold perimetry," Exp Brain Res, vol. 207, no. 3-4, pp. 283-290, Dec. 2010.

[22] K. Matsunaga, M. A. Nitsche, S. Tsuji, and J. C. Rothwell, "Effect of transcranial DC sensorimotor cortex stimulation on somatosensory evoked potentials in humans," Clin Neurophysiol, vol. 115, no. 2, pp. 456-460, Feb. 2004.

[23] R. A. McKinley, L. McIntire, N. Bridges, C. Goodyear, N. B. Bangera, and M. P. Weisend, "Acceleration of image analyst training with transcranial direct current stimulation," Behav. Neurosci., vol. 127, no. 6, pp. 936-946, Dec. 2013.

[24] M. A. Nitsche, K. Fricke, U. Henschke, A. Schlitterlau, D. Liebetanz, N. Lang, S. Henning, F. Tergau, and W. Paulus, "Pharmacological modulation of cortical excitability shifts induced by transcranial direct current stimulation in humans," J. Physiol. (Lond.), vol. 553, no. Pt 1, pp. 293301, Nov. 2003.

[25] M. A. Nitsche, A. Schauenburg, N. Lang, D. Liebetanz, C. Exner, W. Paulus, and F. Tergau, "Facilitation of implicit motor learning by weak transcranial direct current stimulation of the primary motor cortex in the human," J Cogn Neurosci, vol. 15, no. 4, pp. 619-626, May 2003.

[26] G. S. Shekhawat, C. M. Stinear, and G. D. Searchfield, "Transcranial direct current stimulation intensity and duration effects on tinnitus suppression," Neurorehabil Neural Repair, vol. 27, no. 2, pp. 164-172, Feb. 2013.

[27] M. Bikson, J. Dmochowski, and A. Rahman, "The 'quasi-uniform' assumption in animal and computational models of non-invasive electrical stimulation," Brain Stimul, vol. 6, no. 4, pp. 704-705, Jul. 2013.

[28] P. Andersen, T. V. Bliss, and K. K. Skrede, "Unit analysis of hippocampal polulation spikes," Exp Brain Res, vol. 13, no. 2, pp. 208-221, 1971.

[29] E.-H. Park, E. Barreto, B. J. Gluckman, S. J. Schiff, and P. So, "A model of the effects of applied electric fields on neuronal synchronization," Aug. 2005.

[30] G.-S. Yi, J. Wang, X.-L. Wei, K.-M. Tsang, W.-L. Chan, B. Deng, and C.-X. Han, “Exploring how extracellular electric field modulates neuron activity through dynamical analysis of a twocompartment neuron model," Jun. 2014.

[31] J.-Y. Chen, S. Chauvette, S. Skorheim, I. Timofeev, and M. Bazhenov, "Interneuron-mediated inhibition synchronizes neuronal activity during slow oscillation," J. Physiol. (Lond.), vol. 590, no. 16, pp. 3987-4010, Aug. 2012. 
[32] J.-Y. Chen, P. Lonjers, C. Lee, M. Chistiakova, M. Volgushev, and M. Bazhenov,

"Heterosynaptic plasticity prevents runaway synaptic dynamics," J. Neurosci., vol. 33, no. 40, pp. 15915-15929, Oct. 2013.

[33] P. F. Pinsky and J. Rinzel, "Intrinsic and network rhythmogenesis in a reduced Traub model for CA3 neurons," J Comput Neurosci, vol. 1, no. 1-2, pp. 39-60, Jun. 1994.

[34] M. Bazhenov, I. Timofeev, M. Steriade, and T. J. Sejnowski, "Cellular and network models for intrathalamic augmenting responses during 10-Hz stimulation," J. Neurophysiol., vol. 79, no. 5, pp. 2730-2748, May 1998.

[35] P. Poirazi, T. Brannon, and B. W. Mel, "Arithmetic of subthreshold synaptic summation in a model CA1 pyramidal cell," Neuron, vol. 37, no. 6, pp. 977-987, Mar. 2003.

[36] A. R. Brunoni, P. Shiozawa, D. Truong, D. C. Javitt, H. Elkis, F. Fregni, and M. Bikson, "Understanding tDCS effects in schizophrenia: a systematic review of clinical data and an integrated computation modeling analysis," Expert Rev Med Devices, vol. 11, no. 4, pp. 383-394, Jul. 2014.

[37] S. Binder, K. Berg, F. Gasca, B. Lafon, L. C. Parra, J. Born, and L. Marshall, "Transcranial slow oscillation stimulation during sleep enhances memory consolidation in rats," Aug. 2014.

[38] L. Marshall, H. Helgadóttir, M. Mölle, and J. Born, "Boosting slow oscillations during sleep potentiates memory," Nov. 2006.

[39] N. L. Golding and N. Spruston, "Dendritic sodium spikes are variable triggers of axonal action potentials in hippocampal CA1 pyramidal neurons," Neuron, vol. 21, no. 5, pp. 11891200, Nov. 1998.

[40] L. S. Benardo, L. M. Masukawa, and D. A. Prince, "Electrophysiology of isolated hippocampal pyramidal dendrites," J. Neurosci., vol. 2, no. 11, pp. 1614-1622, Nov. 1982.

[41] J. R. Huguenard, O. P. Hamill, and D. A. Prince, "Sodium channels in dendrites of rat cortical pyramidal neurons," Apr. 1989.

[42] W. Regehr, J. S. Kehoe, P. Ascher, and C. Armstrong, "Synaptically triggered action potentials in dendrites," Neuron, vol. 11, no. 1, pp. 145-151, Jul. 1993.

[43] N. Spruston, Y. Schiller, G. Stuart, and B. Sakmann, "Activity-dependent action potential invasion and calcium influx into hippocampal CA1 dendrites," Science, vol. 268, no. 5208, pp. 297-300, Apr. 1995.

[44] R. W. Turner and T. L. Richardson, "Apical dendritic depolarizations and field interactions evoked by stimulation of afferent inputs to rat hippocampal CA1 pyramidal cells," 1991.

[45] R. W. Turner, D. E. Meyers, T. L. Richardson, and J. L. Barker, "The site for initiation of action potential discharge over the somatodendritic axis of rat hippocampal CA1 pyramidal neurons," J. Neurosci., vol. 11, no. 7, pp. 2270-2280, Jul. 1991.

[46] R. Yuste and D. W. Tank, "Dendritic integration in mammalian neurons, a century after Cajal," Neuron, vol. 16, no. 4, pp. 701-716, Apr. 1996.

[47] A. Datta, V. Bansal, J. Diaz, J. Patel, D. Reato, and M. Bikson, "Gyri-precise head model of transcranial direct current stimulation: improved spatial focality using a ring electrode versus conventional rectangular pad," Brain Stimul, vol. 2, no. 4, pp. 201-207, 207.e1, Oct. 2009.

[48] S. Laughlin, "A simple coding procedure enhances a neuron's information capacity," $Z$. Naturforsch., C, Biosci., vol. 36, no. 9-10, pp. 910-912, Oct. 1981.

[49] C. P. Hung, G. Kreiman, T. Poggio, and J. J. DiCarlo, "Fast readout of object identity from macaque inferior temporal cortex," Science, vol. 310, no. 5749, pp. 863-866, Nov. 2005.

[50] C. J. McAdams and J. H. Maunsell, "Effects of attention on orientation-tuning functions of single neurons in macaque cortical area V4," J. Neurosci., vol. 19, no. 1, pp. 431-441, Jan. 1999.

[51] J. H. Reynolds, T. Pasternak, and R. Desimone, "Attention increases sensitivity of V4 neurons," Neuron, vol. 26, no. 3, pp. 703-714, Jun. 2000.

[52] H. Spitzer, R. Desimone, and J. Moran, "Increased attention enhances both behavioral and neuronal performance," Science, vol. 240, no. 4850, pp. 338-340, Apr. 1988. 
[53] M. Bączyk and E. Jankowska, "Presynaptic actions of transcranial and local direct current stimulation in the red nucleus," J. Physiol. (Lond.), vol. 592, no. 19, pp. 4313-4328, Oct. 2014.

[54] F. Bolzoni, M. Bączyk, and E. Jankowska, "Subcortical effects of transcranial direct current stimulation in the rat," J. Physiol. (Lond.), vol. 591, no. 16, pp. 4027-4042, Aug. 2013.

[55] J. Márquez-Ruiz, R. Leal-Campanario, R. Sánchez-Campusano, B. Molaee-Ardekani, F. Wendling, P. C. Miranda, G. Ruffini, A. Gruart, and J. M. Delgado-García, "Transcranial directcurrent stimulation modulates synaptic mechanisms involved in associative learning in behaving rabbits," Apr. 2012.

[56] M. Bikson, M. Inoue, H. Akiyama, J. K. Deans, J. E. Fox, H. Miyakawa, and J. G. R. Jefferys, "Effects of uniform extracellular DC electric fields on excitability in rat hippocampal slices in vitro," May 2004.

[57] J. K. Deans, A. D. Powell, and J. G. R. Jefferys, "Sensitivity of coherent oscillations in rat hippocampus to AC electric fields," J. Physiol. (Lond.), vol. 583, no. Pt 2, pp. 555-565, Sep. 2007.

[58] S. Ozen, A. Sirota, M. A. Belluscio, C. A. Anastassiou, E. Stark, C. Koch, and G. Buzsáki, "Transcranial electric stimulation entrains cortical neuronal populations in rats," J. Neurosci., vol. 30, no. 34, pp. 11476-11485, Aug. 2010.

[59] F. Hummel, P. Celnik, P. Giraux, A. Floel, W.-H. Wu, C. Gerloff, and L. G. Cohen, "Effects of noninvasive cortical stimulation on skilled motor function in chronic stroke," Brain, vol. 128, no. Pt 3, pp. 490-499, Mar. 2005.

[60] M. A. Nitsche and W. Paulus, "Sustained excitability elevations induced by transcranial DC motor cortex stimulation in humans," Neurology, vol. 57, no. 10, pp. 1899-1901, Nov. 2001.

[61] A. Antal, "Excitability Changes Induced in the Human Primary Visual Cortex by Transcranial Direct Current Stimulation: Direct Electrophysiological Evidence," Investigative Ophthalmology \& Visual Science, vol. 45, no. 2, pp. 702-707, Feb. 2004.

[62] K. Kar and B. Krekelberg, "Transcranial electrical stimulation over visual cortex evokes phosphenes with a retinal origin," J. Neurophysiol., vol. 108, no. 8, pp. 2173-2178, Oct. 2012.

[63] M. Carandini, D. J. Heeger, and J. A. Movshon, "Linearity and normalization in simple cells of the macaque primary visual cortex," J. Neurosci., vol. 17, no. 21, pp. 8621-8644, Nov. 1997.

[64] Y. Shu, A. Hasenstaub, M. Badoual, T. Bal, and D. A. McCormick, "Barrages of synaptic activity control the gain and sensitivity of cortical neurons," J. Neurosci., vol. 23, no. 32, pp. 1038810401, Nov. 2003.

[65] P. Dayan and L. F. Abbott, Theoretical neuroscience: computational and mathematical modeling of neural systems. Cambridge, Mass: Massachusetts Institute of Technology Press, 2001.

[66] R. M. Bruno and B. Sakmann, "Cortex is driven by weak but synchronously active thalamocortical synapses," Science, vol. 312, no. 5780, pp. 1622-1627, Jun. 2006.

[67] C. P. Marder and D. V. Buonomano, "Timing and balance of inhibition enhance the effect of long-term potentiation on cell firing," J. Neurosci., vol. 24, no. 40, pp. 8873-8884, Oct. 2004.

[68] F. Pouille and M. Scanziani, "Enforcement of temporal fidelity in pyramidal cells by somatic feed-forward inhibition," Science, vol. 293, no. 5532, pp. 1159-1163, Aug. 2001.

[69] F. Pouille, A. Marin-Burgin, H. Adesnik, B. V. Atallah, and M. Scanziani, "Input normalization by global feedforward inhibition expands cortical dynamic range," Nat. Neurosci., vol. 12, no. 12, pp. 1577-1585, Dec. 2009.

[70] A. R. Gardner-Medwin, "Analysis of potassium dynamics in mammalian brain tissue," $J$. Physiol. (Lond.), vol. 335, pp. 393-426, Feb. 1983.

[71] H. Monai, M. Ohkura, M. Tanaka, Y. Oe, A. Konno, H. Hirai, K. Mikoshiba, S. Itohara, J. Nakai, Y. Iwai, and H. Hirase, "Calcium imaging reveals glial involvement in transcranial direct current stimulation-induced plasticity in mouse brain," Nat Commun, vol. 7, p. 11100, 2016. 
[72] T. Radman, R. L. Ramos, J. C. Brumberg, and M. Bikson, "Role of cortical cell type and morphology in subthreshold and suprathreshold uniform electric field stimulation in vitro," Brain Stimul, vol. 2, no. 4, pp. 215-228, 228.e1-3, Oct. 2009.

[73] J. Reis, H. M. Schambra, L. G. Cohen, E. R. Buch, B. Fritsch, E. Zarahn, P. A. Celnik, and J. W. Krakauer, "Noninvasive cortical stimulation enhances motor skill acquisition over multiple days through an effect on consolidation," Proc. Natl. Acad. Sci. U.S.A., vol. 106, no. 5, pp. 1590 1595, Feb. 2009.

[74] U. Amadi, A. Ilie, H. Johansen-Berg, and C. J. Stagg, "Polarity-specific effects of motor transcranial direct current stimulation on fMRI resting state networks," Neuroimage, vol. 88C, pp. 155-161, Nov. 2013. 


\section{FIGURE CAPTIONS}

Figure 1: Neuronal input-output function under DCS. (A) In-vitro experimental set-up of electrophysiological recordings from rat hippocampal brain slices under DCS. DCS induces a uniform electric field across the slice indicated with the experimentally measured voltage gradient in false color. Dendritic and somatic activities are independently measured in CA1.

Positive/negative field was defined as the positive/negative electrode near stratum pyramidale in CA1 corresponding to the conventional definition of "cathodal" /"anodal" stimulation. Panel A depicts a positive electric field. (B) Population measures. Orthodromic stimulation of presynaptic fibers releases neurotransmitters activating postsynaptic dendrites. Current flows into the population of neurons which can be measured as the fEPSP. This excitatory input induces action potentials in the population of neurons; the aggregation of this spiking activity is recorded as the population spike.(C) Input-output function. Increasing the orthodromic stimulation intensity produces bigger synaptic input measured as the fEPSP (gray traces to the right of the curve) with a corresponding increase in spiking output, measured as the population spike (gray traces on the left of the curve). (D-E) Hypothesis for how DCS may affect the I/O function of a neuron. (D) DCS may affect only synaptic input leaving the I/O function unchanged (control I/O is shown in black). Additionally, DCS may increase synaptic input as indicated by green arrows. The corresponding increase in firing as a direct consequence of the change in synaptic input is depicted as gray arrows. The I/O curve under DCS is shaped by the green points that fall on the original I/O curve. (E) DCS may affect synaptic input and amplify spiking output, shifting the I/O curve. Change in synaptic input is marked with horizontal green arrows; amplification of spiking output due to DCS is marked by the vertical green arrows. The I/O curve under DCS is shifted to the left indicating that neurons are in a more responsive state. 
Figure 2. DCS modulate synaptic input and population firing. (A) Modulation of synaptic efficacy measured in hippocampal slices. fEPSP are significantly modulated $(* \mathrm{p}<0.05)$ by $35 \mathrm{~V} / \mathrm{m}$ DCS. Error bars indicate SEM across slices. Representative traces are shown on the right; blue: -DCS, black: control, red: +DCS. (B) Modulation of recorded population spikes from hippocampal slices. -DCS significantly facilitates population spikes and +DCS inhibits them, but to a lesser extend. Representative traces depict the facilitation effect with -DCS (blue) and inhibition with +DCS (red). (C) Modulation of synaptic input in a single neuron computational model. Excitatory post-synaptic potentials (EPSP), measured at the somatic compartment, are modulated by DCS in the same direction as experimental data. The model is deterministic; therefore there are no error bars. (D) Modulation of population firing during DCS in the neuron computational model. Firing level, measured as the number of neurons that fire during DCS normalized by those during control, are modulated similar to the experimental data. The model is deterministic; therefore there are no error bars. (E) Schematic of two-compartment model. The dendritic compartment is formed by a leaky conductance $\left(g_{d l}\right)$ and a capacitive current; voltage dependent conductances including: potassium $\left(g_{\mathrm{K}}\right)$, sodium $\left(g_{\mathrm{Na}}\right)$, persistent sodium $\left(g_{\mathrm{Nap}}\right)$ and slow voltage-dependent non-inactivating potassium $\left(g_{\mathrm{Ks}}\right)$. These are connected through a coupling conductance $\left(g_{\mathrm{C}}\right)$ to the axon/soma compartment including a potassium $\left(g_{\mathrm{K}}\right)$, sodium $\left(g_{\mathrm{NA}}\right)$ and a persistent sodium $\left(g_{\mathrm{Nap}}\right)$ conductance in addition to a leaky current $\left(g_{\mathrm{sl}}\right)$ and a capacitive current. The extracellular electric field was modeled as a voltage difference $\left(V_{\mathrm{E}}\right)$ across the compartments. The voltage traces on the right, show the membrane polarization induced in each compartment by +DCS and -DCS. The coupling constant (amount of polarization induced by each $\mathrm{V} / \mathrm{m}$ ) for the somatic voltage during -DCS is 0.12 $\mathrm{mV} / \mathrm{V} / \mathrm{m}$ and $-0.11 \mathrm{mV} / \mathrm{V} / \mathrm{m}$ for $+\mathrm{DCS}$. In the dendritic compartment the coupling constant is 0.013 $\mathrm{mV} / \mathrm{V} / \mathrm{m}$ for + DCS and $-0.08 \mathrm{mV} / \mathrm{V} / \mathrm{m}$ for -DCS. (F) Synaptic efficacy modulation is independent of orthodromic stimulation orientation. Left, schematic of the experimental set-up, the bipolar stimulation electrode was placed in the middle of CA1. One recording electrode was located closer 
to CA3 (proximal location, to the left of the bipolar stimulation electrode), and the other was placed to the right of the stimulation electrode (distal location). The relative change of the fEPSP slope caused by the field in the proximal location versus the change in the distal location is shown.

Figure 3. DCS modulates the threshold of the neuronal input-output function. (A) Model prediction of the DCS effect on the input-output function. Spiking output is characterized by the probability of firing and synaptic input by the average EPSP slope magnitude measured at the soma. The direction of the field relative to the model is plotted in the upper inset, -DCS (blue) and +DCS (red). (B) Neuronal input-output function is affected by DCS in hippocampal brain slices. Spiking output is measured as the population spike amplitude (PS) and synaptic input as the fEPSP slope. The I/O curve of a representative slice shows the threshold shift $\left(\mathrm{X}_{50}\right)$ induced by $-35 \mathrm{~V} / \mathrm{m}$ DCS (blue) and a smaller change in the opposite direction for $+35 \mathrm{~V} / \mathrm{m}$ DCS (red). Dashed lines mark the $\mathrm{X}_{50}$ value for each curve. Gray area depicts the fEPSP slopes and PS values plotted on (D). The direction of the electric field relative to the slice is plotted in the upper inset. (C) Threshold changes predicted by model and measured in experimental data. Percentage changes are quantified by normalizing the parameters in the field condition by the control condition and calculating the deviation. Gray points indicate the change for each slice; black lines on top of the bars indicate standard error. Note that the model yields one neuronal input-output function in each condition; therefore there are no error bars for the model parameters. (D) DCS affects population firing for an equal synaptic input. Values within the I/O curve with the same synaptic input were chosen (gray area in B), fEPSP traces are plotted on the left and population spikes on the right column for each condition: control (black), DCS (blue), +DCS (red). The three conditions are overlaid in the lower plot. 
Figure 4. DCS alters firing time. (A) Time delay determined by the two-compartment model. Left, representative voltage traces of single spike timing, at determined pre-synaptic stimulation intensity. Time delay $(\Delta \mathrm{T})$ is calculated as the spike latency in control condition (black) minus the time of the action potential during DCS (-DCS: blue, +DCS: red). During -DCS neurons fire faster, resulting in a negative $\Delta \mathrm{T}$; the opposite occurs for +DCS. Right, $\Delta \mathrm{T}$ was measured for both polarities under different orthodromic stimulation intensities (to emulate experimental data). -DCS advances firing time for all orthodromic stimulation intensities (B) Time delay quantified for experimental data. Left, representative physiological traces of population firing with fix orthodromic stimulation intensity. $\Delta \mathrm{T}$ is measured comparing population spike latency (short line underneath each trace) under control versus DCS condition. The population of neurons fire faster during -DCS (blue) and viceversa for + DCS (red). Right, $\Delta \mathrm{T}$ measured across orthodromic stimulation intensities for each slice ( $\mathrm{n}=15)$. -DCS significantly advances population firing and +DCS delays firing. Gray dots indicate the mean $\Delta \mathrm{T}$ across orthodromic stimulation intensities for each slice. Lines on top of the bars indicate standard error.

Figure 5. I/O function model for population of neurons predicts different excitability changes under the anode versus cathode. (A) Single-neuron model predicts modulation for various field intensities and orientations. (A.1) Horizontal shift of the I/O function for multiple field magnitudes. Orientation of the field is aligned with the somato-dendritic axis. The computational model predicts a monotonic modulation (approximately linear) of the horizontal shift of the I/O curve ( $\mathrm{x}_{50}$ shift). Sensitivity to the field intensity varied between positive and negative fields. Resulting in an approximate change of $-0.45 \%$ of the $\mathrm{X}_{50}$ compared to control per $\mathrm{V} / \mathrm{m}$ applied for negative fields and $0.04 \%$ shift per $\mathrm{V} / \mathrm{m}$ for positive fields. (1) and (2) indicate the change of the $\mathrm{I} / \mathrm{O}$ function shown in A.2.(A.2-A.4) For a fixed field amplitude $(35 \mathrm{~V} / \mathrm{m})$ the relative orientation was varied from $0^{\circ}$ (A.2) to $45^{\circ}$ (A.3) to $90^{\circ}$ (A.4), inset indicates direction and polarity of the field. (B) The 
single-neuron model was coupled with a multi-scale model of macroscopic currents in the human brain (Rahman, 2013). We simulated the effect of polarizing currents on the I/O function for neurons distributed across the cortex under the anode and cathode. (B.1) A conventional M1-SO montage was simulated. (B.2) The current flow intensity alternated regularly across the cortex (false color map shows the electric field along the somato-dendritic axis) (B.3) Schematic of the cortical gyrius and the neuronal orientation relative to the current flow. Gray arrows depict the direction of the current. The polarization effect on the I/O function is shown for each group on neurons in the gyrius (I/O functions plotted outside the schematic gyrius). The aggregate effect is calculated as the average across the population of neurons (I/O curve in the center of the gyrius). When current flows inward neurons experience a soma depolarization (blue) resulting in a larger shift of the I/O function to the left. The opposite effect occurs for neurons on the contralateral area of the gyrus. (B.4) Probability density function of the radial component of the electric field underneath the anode and the cathode. The gradient indicates the type of cell body polarization (blue, depolarizing; red, hyperpolarizing). (a) and (c) refer to the $5^{\text {th }}$ percentile; (b) and (d) refer to the $95^{\text {th }}$ percentile. (B.5) Schematic representation of the mean effect on the I/O function of all neurons that are exposed the electric field distribution in (B.4). Upper, the population effect under the anode is a leftward shift shown in blue. Lower, the population effect under the cathode is a rightward shift, plotted in red. (B.6) I/O function change for the population underneath the anode and the cathode quantified as $\%$ change of the $\mathrm{X}_{50}$ threshold per $\mathrm{V} / \mathrm{m}$ applied. (B.7) I/O function modulation for the population of neurons that experience the peak electric fields under the electrodes. $(a, c) 5 \%$ of neurons that are exposed to the strongest negative fields (soma depolarizing) under the anode. (b,d) $5 \%$ of neurons affected by the strongest positive fields (soma hyperpolarizing) under the anode/cathode. 
A Hippocampal slices
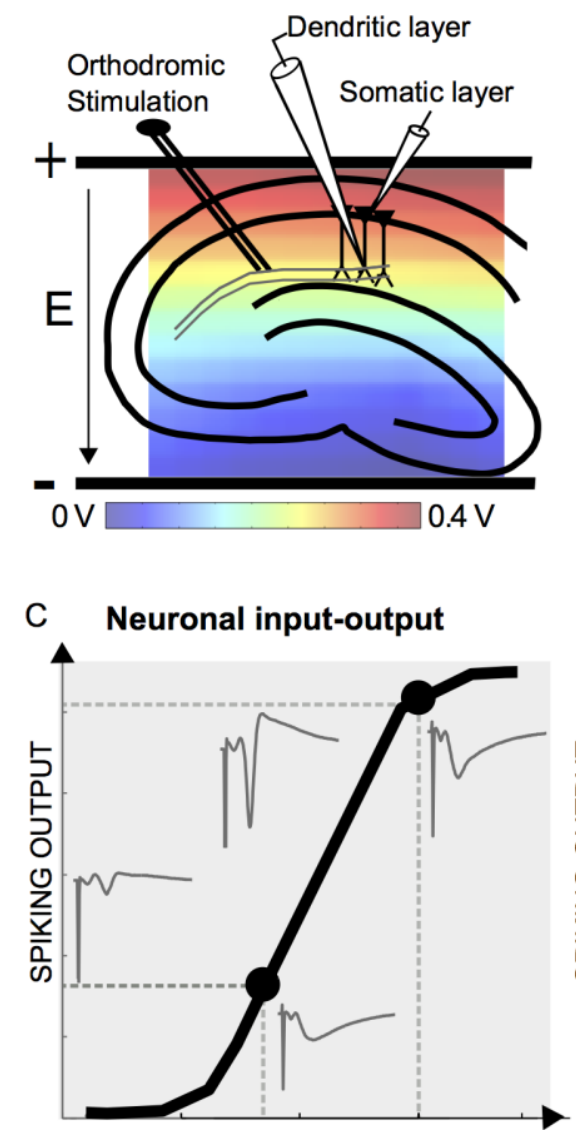

SYNAPTIC INPUT
B
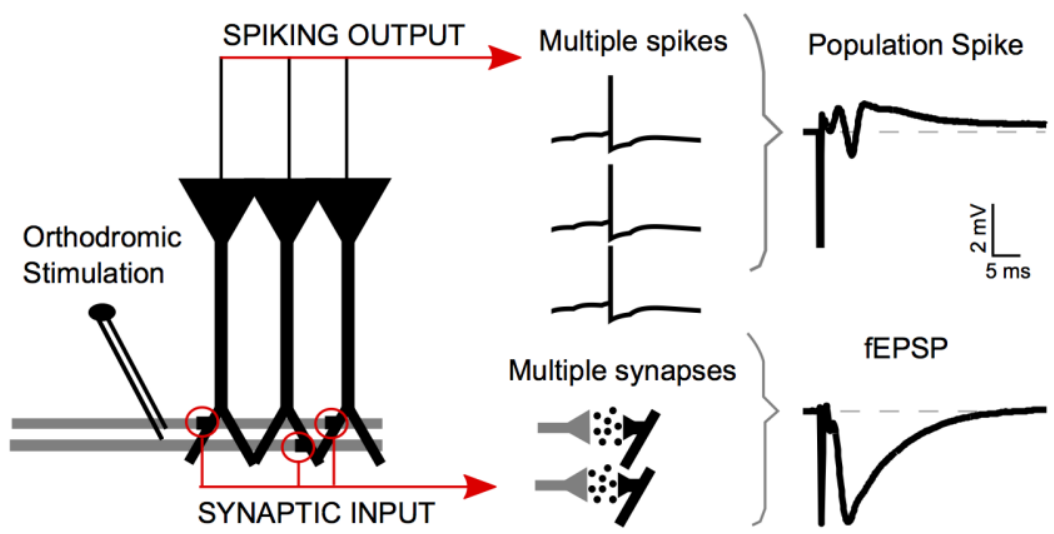

Hypothesis
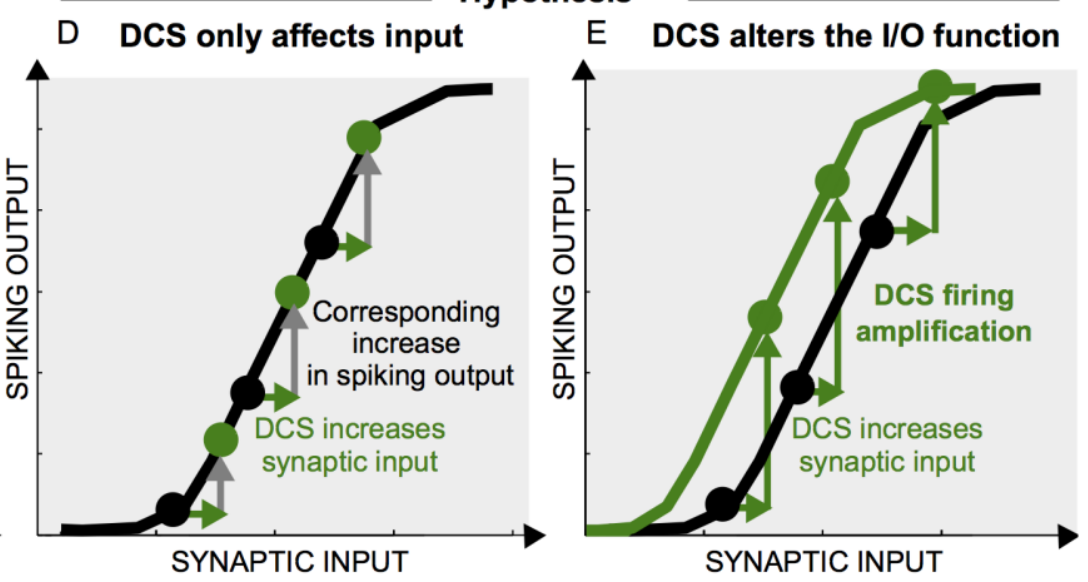

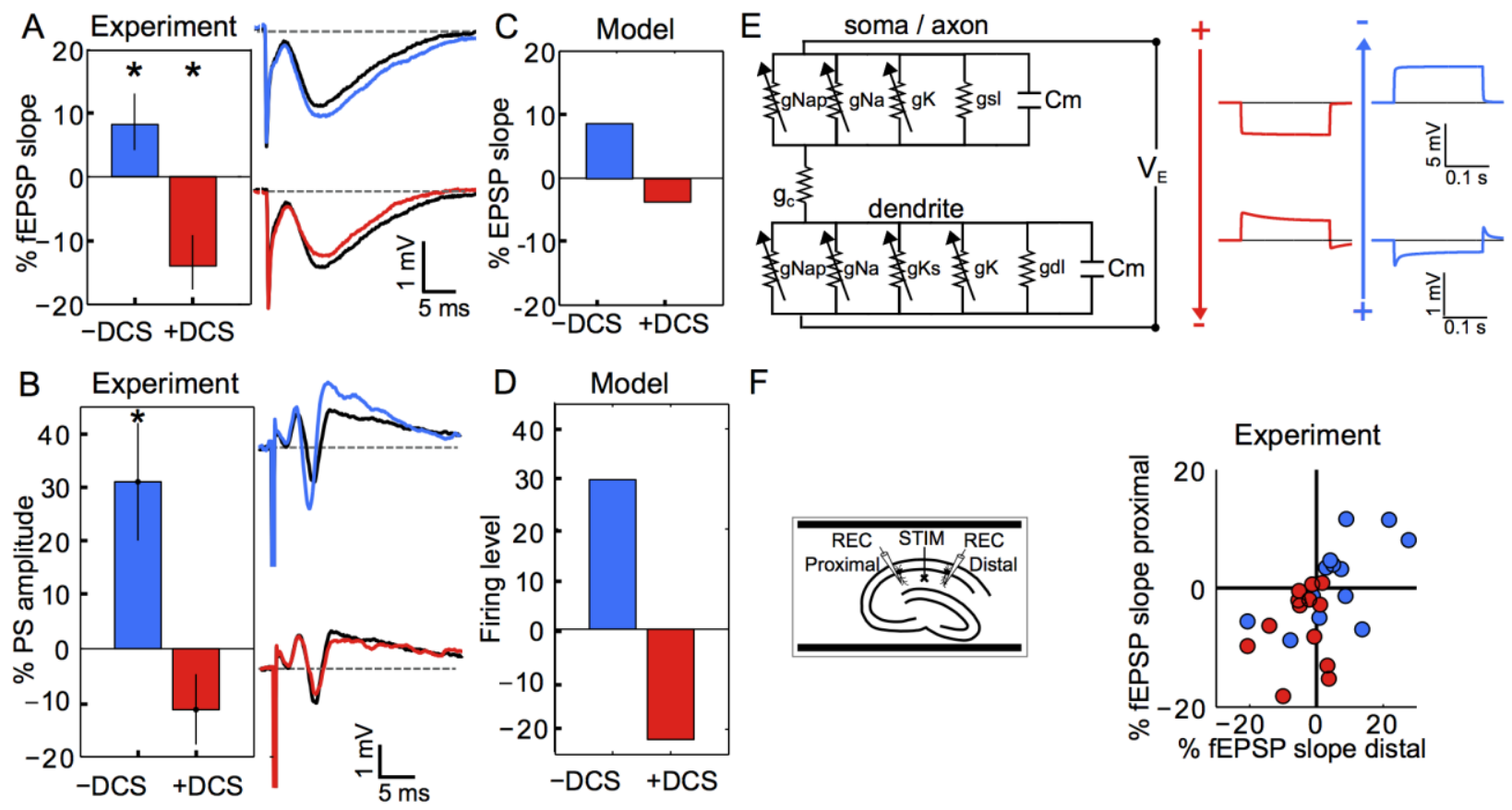


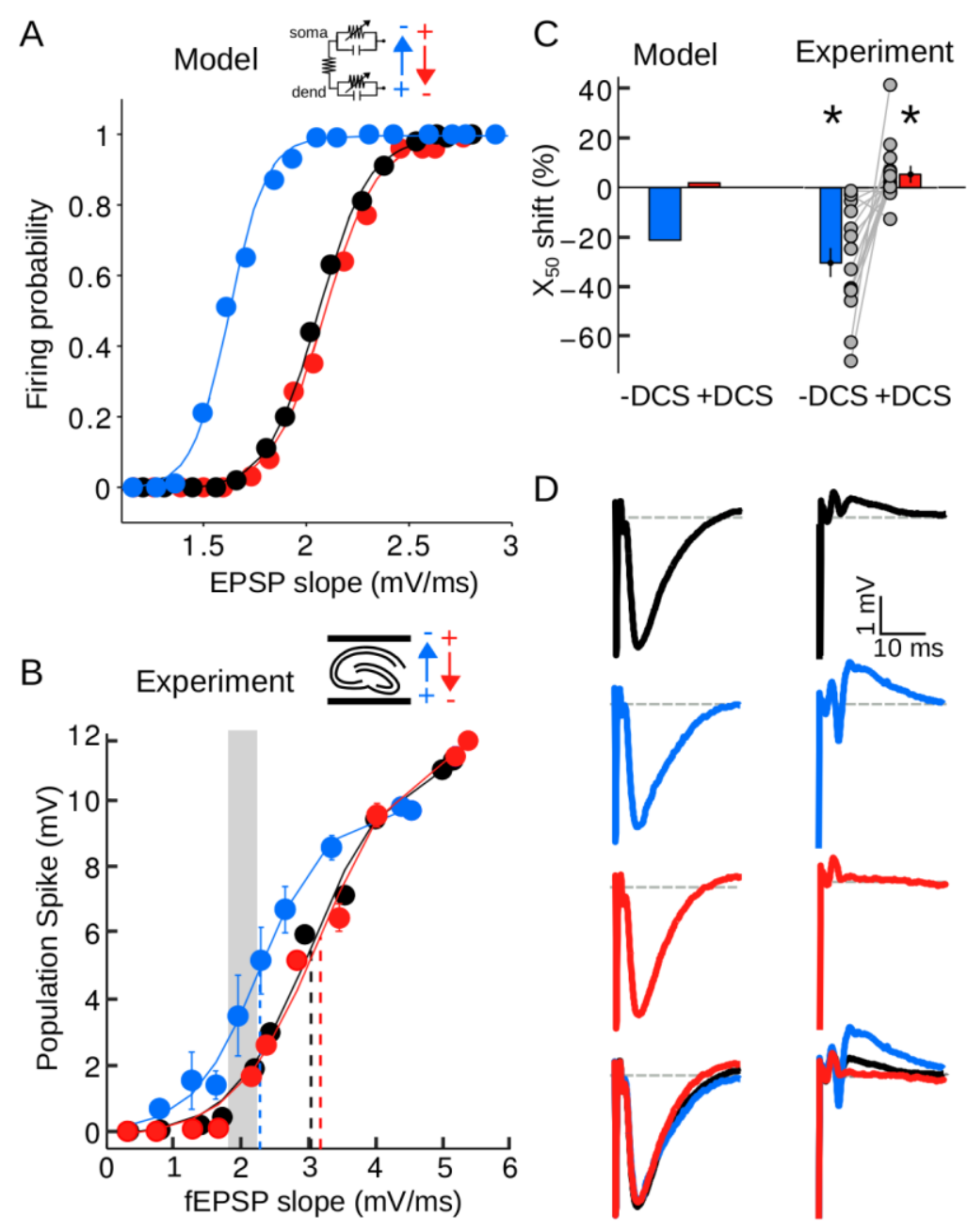



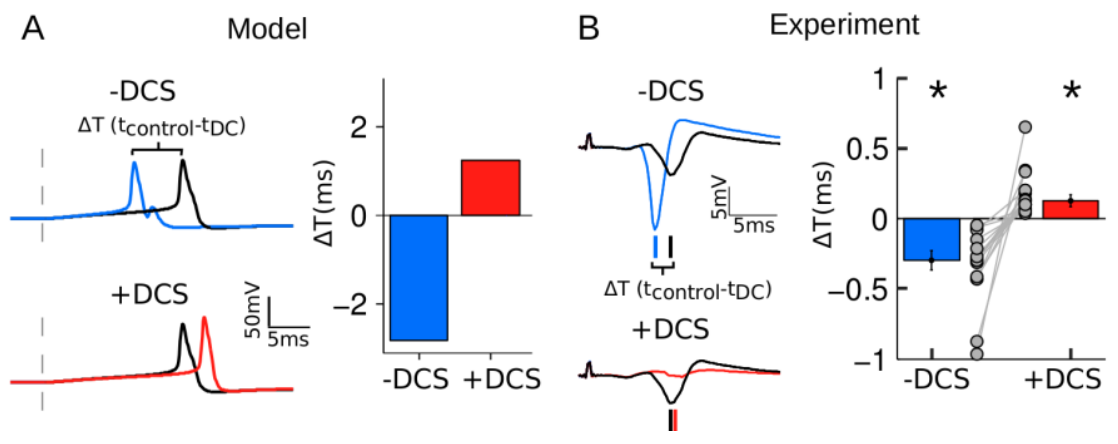
A I/O changes in a single-neuron model by intensity and orientation of DCS
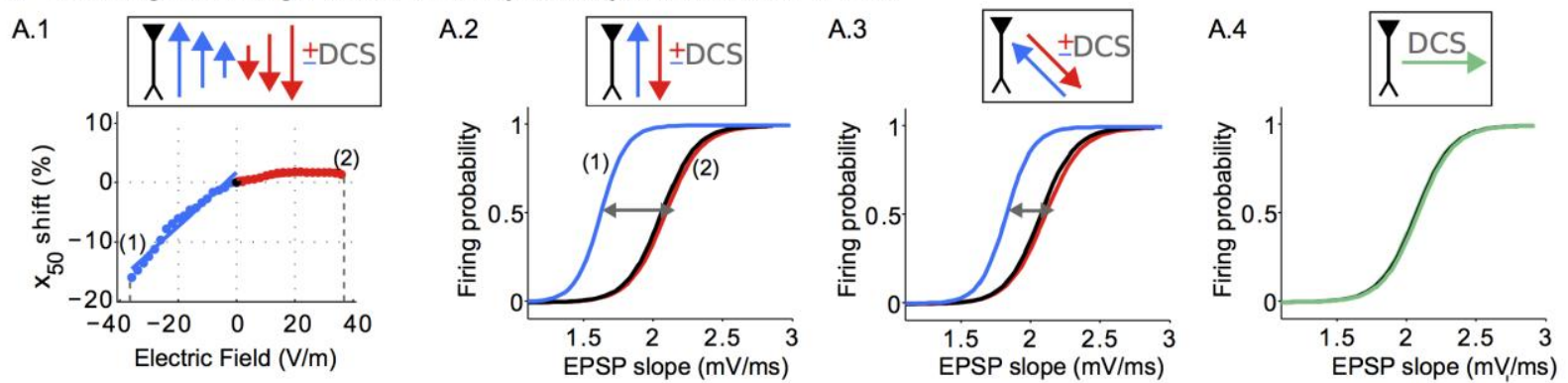

B I/O changes in human cortex during tDCS

B.1

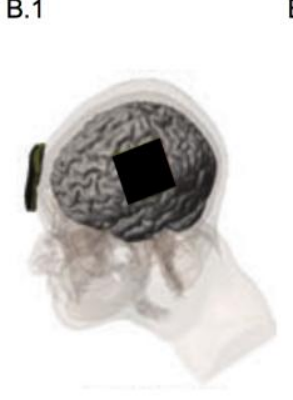

B.2

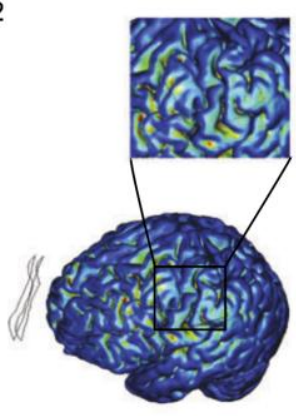

B.4
Area under

$$
\text { the }
$$
ANODE

(a)

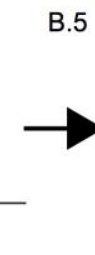

B.5
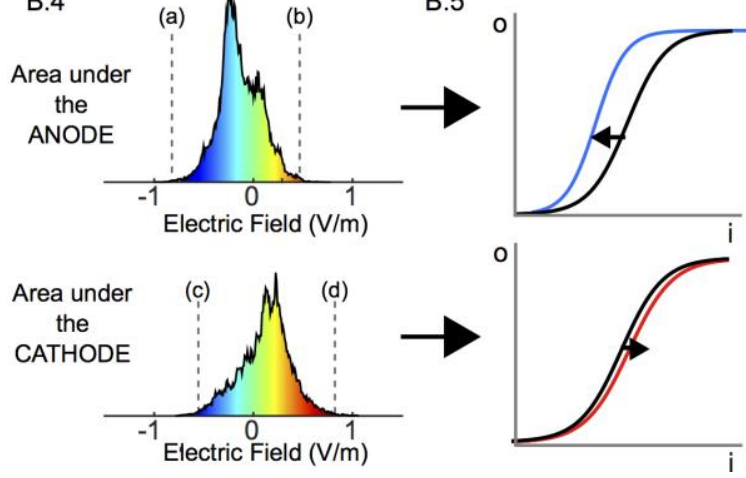

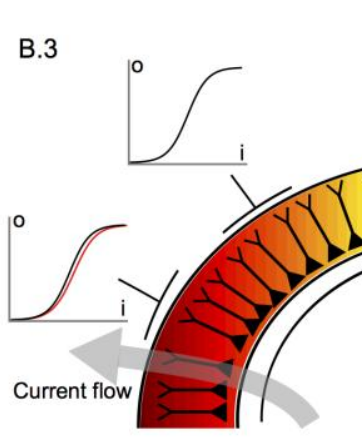

B.6

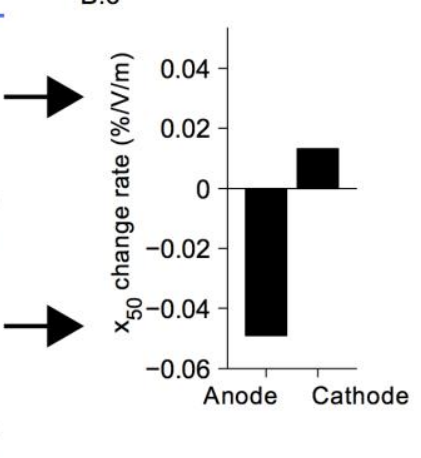

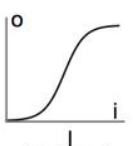
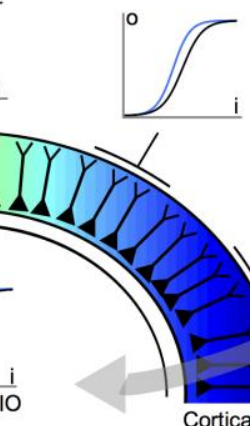

B.7

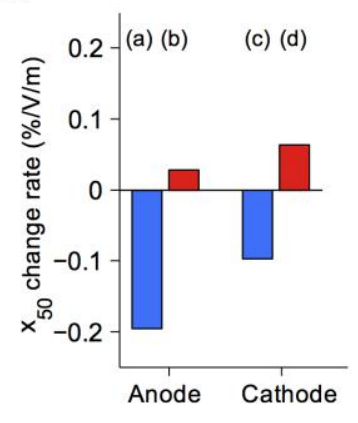

